Original article

\title{
Alendronate and Raloxifene Therapy in the Early Period after Hip Fracture
}

\author{
Tsuyoshi Ohishi ${ }^{1}$, Tetsuya Ichikawa ${ }^{2}$, Takanori Ito $^{3}$, Hiroshi Koyama ${ }^{3}$, Michihito Miyagi ${ }^{3}$, \\ Hironobu Hoshino ${ }^{3}$ and Masaaki Takahashi ${ }^{3}$ \\ ${ }^{1}$ Department of Orthopaedic Surgery, Enshu Hospital, Japan \\ ${ }^{2}$ Department of Orthopaedic Surgery, Narita Memorial Hospital, Japan \\ ${ }^{3}$ Department of Orthopaedic Surgery, Hamamatsu University School of Medicine, Japan
}

\begin{abstract}
Objective: The purpose of the present study was to clarify the efficacy of alendronate and raloxifene for preventing bone loss in patients with hip fracture by monitoring bone mineral densities (BMDs) and biochemical markers during the 9-month period after fracture.

Patients and Methods: Eighty-two female hip fracture patients from 50 to 99 years old (mean \pm SD: $81.6 \pm 9.5$ ) were randomly divided into two groups; there were 46 patients in the alendronatetreated group (group ALN) and 36 patients in the raloxifene-treated group (group RLX). Drugs were administered to patients six weeks after their operations. Lumbar spine BMD and neck, trochanter, Ward's and total BMDs of the contralateral proximal femur, serum intact osteocalcin (intact OC), bone-specific alkaline phosphatase (BAP) and urinary N-terminal telopeptide of type I collagen (NTX) were measured just before the start of drug administration and at 9 months thereafter.

Results: Twenty-two out of 46 patients in group ALN and 23 out of 36 patients in group RLX completed the study. The most common reason for dropping out was the patient's failure to visit the outpatient clinic. Trochanter BMD in group ALN tended to increase by $8.4 \%$ compared with the baseline, and total hip BMD in group RLX showed a significant increase (5.7\%), although neck BMD in both groups decreased during the 9 months of treatment $(-8.7 \%$ for group ALN and $-4.2 \%$ for group RLX compared with the baseline). Spine BMD did not change significantly in eithr group. Serum BAP and urinary NTX decreased significantly in both groups. Serum intact OC did not change significantly.

Conclusions: Both alendronate and raloxifene have a favorable effect on trochanter and total BMDs of the contralateral proximal femur in the short period after hip fracture. However, both drugs could not prevent bone loss in the femoral neck during the 9 months of treatment.
\end{abstract}

Correspondence to: Tsuyoshi Ohishi, Department of Orthopaedic

Surgery, Enshu Hospital, 1-1-1 Chuo, Naka-ku, Hamamatsu, Shizuoka 430-0929, Japan

E-mail address: t-ohishi@ken.ja-shizuoka.or.jp
Key words: alendronate, bone mineral density, dropout, hip fracture, raloxifene

(J Rural Med 2011; 6(1): 16-21)

\section{Introduction}

Bone loss following hip fracture is severe even if patients can walk in the early period after their operations. As a result, it is not uncommon for patients who suffer a hip fracture to experience a second hip fracture. Indeed, the risk of suffering a second hip fracture is sixfold greater than the risk of initial hip fracture ${ }^{1}$. Among patients with bilateral hip fractures, thirty-eight percent of second hip fractures occur less than 1 year after the initial fracture ${ }^{2)}$. Prevention of excess bone loss within one year after hip fracture is therefore essential to prevent subsequent occurrence of fragility fractures. Alendronate and raloxifene are both firstline drugs recommended in the guidelines for osteoporosis treatment ${ }^{3)}$. Alendronate reduced the risk of new vertebral and non-vertebral fractures including hip fractures ${ }^{4-6)}$. Raloxifene decreased new vertebral fracture risk and also nonvertebral fracture risk in a subgroup of women with severe vertebral fractures at baseline ${ }^{7-9)}$. Therefore, both alendronate and raloxifene may be expected to prevent bone loss after hip fracture and prevent subsequent fragility fractures. According to the guidelines for osteoporosis treatment, patients with a hip fracture are candidates for treatment with anti-osteoporosis drugs ${ }^{3)}$. In recent years, the number of hip fracture patients receiving treatment with anti-osteoporosis drugs has been increasing ${ }^{10,11)}$. However, many patients are still not receiving this treatment ${ }^{12-15)}$. In the present study, we administered alendronate or raloxifene for 9 months to patients with hip fractures in the early period after operation and monitored bone mineral densities (BMDs) and biochemical markers. The primary goal of this study was to investigate the effect of alendronate or raloxifene on BMD 
and biochemical markers. The secondary goal was to investigate the reasons for dropping out during the study.

\section{Patients and Methods}

Eighty-two female patients with hip fractures from 50 to 99 years old (mean \pm SD: $81.6 \pm 9.5$ ) were recruited at Enshu Hospital (Hamamatsu, Shizuoka, Japan) between 2005 and 2007. All subjects suffered from hip fractures resulting from a simple fall from standing height or lower and were admitted to our hospital within 24 hours after incidence of the fracture. Within 3 days after admission, cementless hemiarthroplasty (Kyocera PerFix) or osteosynthesis with three $6.5-\mathrm{mm}$ cannulated cancellous screws (Ace Medical) was used to treated femoral neck fractures, and osteosynthesis with compression hip screws (Ace Medical) or $\gamma$-nails (Howmedica) was used to treated trochanteric fractures. All subjects were allowed to walk 3 days after surgery. Patients were excluded if they met any of the following criteria in the two years before the fracture: 1) were admitted with a pathologic fracture, 2) had sustained a previous fracture, 3) had a thyroid disorder, 4) had suffered from alcohol abuse, 5) had taken calcium, vitamin $D$, bisphosphonate, vitamin $\mathrm{K}$, thiazide or glucocorticoids supplements, 6) had suffered from osteomalacia after gastrectomy, 7) had undergone an orchidectomy, 8) were receiving hormone replacement therapy for prostate disease, 9) were unable to walk or 10) had pseudoarthrosis of the fracture site and osteonecrosis of the femoral head. Informed consent was obtained from each patient prior to participation in the study. The patients were randomly divided into two groups. Group ALN consisted of 46 patients treated orally with $5 \mathrm{mg}$ daily doses of alendronate on an empty stomach. Group RLX consisted of 36 patients treated orally with $60 \mathrm{mg}$ daily doses of raloxifene after breakfast. Drugs were administered for 9 months beginning 6 weeks after the operation. Blood and spot urine samples were obtained at 9:00 to 11:00 $\mathrm{AM}$ before drug administration and 9 months after administration. Bone mineral densities were measured before administration and 9 months after administration using dual-energy X-ray absorptiometry (DXA) from a QDR Discovery scanner (Hologic, Inc. Madison, Bedford, MA, U.S.A.). Bone mineral density in grams per square centimeter was determined for the lumbar spine (L2-4 in anteroposterior projection) and four regions (femoral neck, trochanter, Ward's triangle and total hip) in the proximal femur of the contralateral side. The precision of the integral calcium hydroxyapatite standard was less than $1.0 \%$ over time. Serum levels of intact osteocalcin (intact OC), bone specific alkaline phosphatase (BAP) and urinary levels of cross-linked N-terminal telopeptides of type I collagen (NTX) were measured. Serum intact OC was measured by sandwich enzyme-linked immunosorbent assay using a commercially available kit (Intact Osteocalcin, Biomedical Technologies Inc., Stoughton, MA, USA) with intra- and interassay variations of $7 \%$ and $10.5 \%$, respectively. Serum BAP was measured by enzymelinked immunosorbent assay with intra- and interassay variations of $3.9-5.9 \%$ and $4.4-7.7 \%$, respectively ${ }^{16}$. The urinary levels of NTX were measured with an enzymelinked immunosorbent assay with an intra-assay coefficient of variation was $7 \%{ }^{17}$. The NTx concentration was normalized to urinary creatinine.

\section{Statistical Analysis}

Data were analyzed using a StatView 5.0 (SAS Institute, Cary, NC, USA) program on a Macintosh computer. A non-parametric test, followed by a Mann-Whitney U test was used for comparison of the data between groups. Comparison of two related values within groups was analyzed by a Wilcoxon signed-rank test. All values except those of biochemical parameters were expressed as means \pm standard deviation (SD). Values of biochemical parameters were expressed as means \pm standard error (SE). Statistical significance was defined as less than 0.05 .

\section{Results}

Twenty-two out of 46 patients in group ALN and 23 out of 36 patients in group RLX completed the study. Significant differences were not observed between group ALN and group RLX in anthropometric, BMDs and biochemical parameters at baseline (Tables 1, 2 and 3). Table 2 shows values of BMDs at baseline and 9 months after drug administration. Lumbar spine BMD in both groups did not change significantly. Although neck BMD decreased significantly, trochanter BMD tended to increase in group ALN. Ward's BMD did not change significantly in either group. Total BMD in the proximal femur in group RLX increased significantly. Percent changes in three regions of the proximal femur compared with the BMD values before drug administration in each group are also indicated in Table 2. In both groups, trochanter and total BMDs tended to increase; however, neck BMD tended to decrease in spite of the antiosteoporosis drug therapy.

Serum BAP and urinary NTX decreased significantly compared with the baseline values in both groups (Table 3). In particular, urinary NTX in group ALN decreased by $55.0 \%$ compared with the value before drug administration. Serum intact OC did not change in either group. All patients who completed the study could walk with a single cane or walking trolley during the study period. For the pa- 
Table 1 Anthropometric data for group ALN and group RLX

\begin{tabular}{lcc}
\hline & Group ALN & Group RLX \\
\hline $\mathrm{N}$ & 22 & 23 \\
Age (years) & $79.4 \pm 10.7$ & $81.3 \pm 6.5$ \\
Height $(\mathrm{cm})$ & $146.9 \pm 7.3$ & $146.4 \pm 6.6$ \\
Weight $(\mathrm{kg})$ & $42.8 \pm 9.8$ & $45.7 \pm 9.1$ \\
BMI $\left(\mathrm{kg} / \mathrm{m}^{2}\right)$ & $19.7 \pm 3.4$ & $21.2 \pm 3.5$ \\
Trochanteric fracture $(\mathrm{N}) /$ Neck fracture $(\mathrm{N})$ & $5 / 17$ & $6 / 17$ \\
Osteosynthesis $(\mathrm{N}) /$ Endoprosthesis $(\mathrm{N})$ & $10 / 12$ & $11 / 12$ \\
\hline
\end{tabular}

Data are shown as means $\pm \mathrm{SD}$.

BMI: body mass index.

Table 2 Changes in bone mineral density in group ALN and group RLX

\begin{tabular}{|c|c|c|c|c|}
\hline & Baseline & After therapy & Percent change & P-values \\
\hline \multicolumn{5}{|c|}{ Lumbar spine BMD $\left(\mathrm{g} / \mathrm{cm}^{2}\right)$} \\
\hline Group ALN & $0.648 \pm 0.160$ & $0.650 \pm 0.139$ & $1.0 \pm 6.0 \%$ & 0.476 \\
\hline Group RLX & $0.737 \pm 0.171$ & $0.701 \pm 0.195$ & $-4.7 \pm 15.5 \%$ & 0.370 \\
\hline \multicolumn{5}{|c|}{ Neck BMD $\left(\mathrm{g} / \mathrm{cm}^{2}\right)$} \\
\hline Group ALN & $0.455 \pm 0.087$ & $0.415 \pm 0.089$ & $-8.7 \pm 8.9 \%$ & 0.0008 \\
\hline Group RLX & $0.465 \pm 0.138$ & $0.430 \pm 0.107$ & $-4.2 \pm 15.4 \%$ & 0.089 \\
\hline \multicolumn{5}{|c|}{ Trochanter BMD $\left(\mathrm{g} / \mathrm{cm}^{2}\right)$} \\
\hline Group ALN & $0.352 \pm 0.096$ & $0.382 \pm 0.095$ & $8.4 \pm 17.2 \%$ & 0.067 \\
\hline Group RLX & $0.384 \pm 0.123$ & $0.398 \pm 0.114$ & $7.8 \pm 18.4 \%$ & 0.131 \\
\hline \multicolumn{5}{|c|}{ Ward's triangle BMD $\left(\mathrm{g} / \mathrm{cm}^{2}\right)$} \\
\hline Group ALN & $0.261 \pm 0.089$ & $0.266 \pm 0.081$ & $4.2 \pm 31.7 \%$ & 0.728 \\
\hline Group RLX & $0.297 \pm 0.157$ & $0.275 \pm 0.129$ & $11.1 \pm 63.2 \%$ & 0.654 \\
\hline \multicolumn{5}{|c|}{ Total hip BMD $\left(\mathrm{g} / \mathrm{cm}^{2}\right)$} \\
\hline Group ALN & $0.480 \pm 0.106$ & $0.500 \pm 0.110$ & $3.8 \pm 11.9 \%$ & 0.131 \\
\hline Group RLX & $0.508 \pm 0.143$ & $0.527 \pm 0.146$ & $5.7 \pm 10.8 \%$ & 0.030 \\
\hline
\end{tabular}

Data are shown as means \pm SD. BMD: bone mineral density.

tients who completed the study, none of the X-rays showed peseudoarthrosis or osteonecrosis of the proximal femur or loosening of endoprosthesis at the end of the study.

As for the dropout rate, 24 patients in group ALN and 13 patients in group RLX failed to complete the study. The reasons for dropping out in each group are listed in Table 4. Two patients in group ALN and one patient in group RLX were withdrawn because they suffered new fragile fractures during the study. The most common reason for dropping out was failure to visit the outpatient clinic. Only one patient in group RLX discontinued drug treatment because of hot flashes, an adverse effect of the drug. One patient experienc- ing hot flashes overcame the side effects after cessation of the therapy.

\section{Discussion}

In the present study, neck BMD of the proximal femur in group ALN and in group RLX decreased significantly, by $8.7 \%$ and $4.2 \%$ after 9 months of treatment, respectively. Neck BMD after hip fracture decreased by $4.3-9.2 \%$ without anti-osteoporosis drug intervention of the course of one year in previous papers ${ }^{18-22}$. Surprisingly, neck BMD decreased by more compared with the percentage changes in 
Table 3 Changes in biochemical markers in group ALN and group RLX

\begin{tabular}{|c|c|c|c|c|}
\hline & Baseline & After therapy & Percent change & Reference range \\
\hline Serum intact $\mathrm{OC}(\mathrm{ng} / \mathrm{ml})$ & & & & $2-7$ \\
\hline Group ALN & $2.78 \pm 0.45$ & $2.02 \pm 0.32$ & $-4.3 \pm 20.0 \%$ & \\
\hline Group RLX & $3.24 \pm 0.55$ & $3.04 \pm 0.61$ & $11.8 \pm 24.2 \%$ & \\
\hline Serum BAP (U/L) & & & & $9.6-35.4$ \\
\hline Group ALN & $38.5 \pm 2.6$ & $27.8 \pm 2.5^{* *}$ & $-19.2 \pm 12.4 \%$ & \\
\hline Group RLX & $44.5 \pm 3.2$ & $34.4 \pm 2.6^{* *}$ & $-20.0 \pm 5.7 \%$ & \\
\hline Urinary NTX (nmol BCE/mmol Cr) & & & & $14.3-89.0$ \\
\hline Group ALN & $102.2 \pm 11.2$ & $38.4 \pm 5.4^{* * *}$ & $-55.0 \pm 8.5 \%$ & \\
\hline Group RLX & $106.8 \pm 11.7$ & $51.2 \pm 9.4^{* * *}$ & $-47.1 \pm 9.8 \%$ & \\
\hline
\end{tabular}

Values of biochemical markers and percent changes are shown as means \pm SE. OC: osteocalcin. BAP: bone-specific alkaline phosphatase. NTX: N-terminal telopeptides of type I collagen. BCE: bone collagen equivalents of NTX immunoreactivity.

** and $* * * \mathrm{p}<0.01$ and $\mathrm{p}<0.001$ compared with the baseline values, respectively.

Table 4 Reasons for dropping out from the study

\begin{tabular}{ll}
\hline \multicolumn{1}{c}{ Group ALN $(\mathrm{n}=24)$} & \multicolumn{1}{c}{ Group RLX $(\mathrm{n}=13)$} \\
\hline New contra-lateral hip fracture $(\mathrm{n}=1)$ & New contra-lateral hip fracture $(\mathrm{n}=1)$ \\
New vertebral fracture $(\mathrm{n}=1)$ & Refracture $(\mathrm{n}=1)$ \\
Device failure $(\mathrm{n}=1)$ & Hot flashes $(\mathrm{n}=1)$ \\
Death $(\mathrm{n}=1)$ & Heart failure $(\mathrm{n}=1)$ \\
Failure to visit clinic $(\mathrm{n}=8)$ & Failure to visit clinic $(\mathrm{n}=5)$ \\
Transfer to other hospital $(\mathrm{n}=3)$ & Urine could not be collected $(\mathrm{n}=1)$ \\
Urine could not be collected $(\mathrm{n}=2)$ & Others $(\mathrm{n}=3)$ \\
Others $(\mathrm{n}=7)$ & \\
\hline
\end{tabular}

past studies even when alendronate or raloxifene was administrated. This may partly be due to neck BMD at the baseline in the present study being lower than that in past studies. In the present study, the mean values of neck BMD at the baseline in the two groups were 0.455 and $0.465 \mathrm{~g} /$ $\mathrm{cm}^{3}$; however, those in two past studies were 0.559 and $0.546 \mathrm{~g} / \mathrm{cm}^{3}$, respectively ${ }^{19,20)}$. Trochanter BMD in group ALN tended to increase, and total BMD of the proximal femur in group RLX increased significantly with the 9 months of anti-osteoporosis drug intervention. Our results are in good agreement with those reported by Cecilia et al. ${ }^{23)}$. They investigated antiresorptive efficacy on spine and hip BMDs of alendronate given immediately after operation in hip fracture patients. Total, trochanteric and intertrochanteric BMDs of the hip increased significantly after alendronate therapy, while neck BMD did not change significantly according to their results. In our previous study, vitamin $\mathrm{K}_{2}$ prevented bone loss in the Ward's triangle of the contralateral proximal femur after hip fracture over the course of 9 months ${ }^{24)}$. The anti-osteoporosis effect of alendronate, raloxifene and vitamin $\mathrm{K}_{2}$ may first impact cancellous bone rather than cortical bone in the short term. Trochanter, total and Ward's BMDs reflect cancellous bone predominantly; however, neck BMD reflects mainly cortical bone. Therefore, the favorable effects of alendronate and raloxifene were reflected in trochanter and total BMDs rather than neck BMD in the short period of this study.

In both groups, serum BAP and urinary NTX decreased significantly during the study period; however, serum intact OC did not change significantly. Bone resorption markers increased rapidly after hip fracture, with peak values at 4 to 8 weeks after fracture, and decreased thereafter up to 12 months, while bone formation markers increased gradually after fracture up to 12 months $^{25}$. In this study, bone resorption marker or NTX decreased significantly compared with the baseline values during the 9 months because of the strong antiresoptive effect of the drugs. Serum bone formation marker or BAP decreased during the 9 months because 
suppression of bone turnover by the drugs might mask the increased rate of bone formation in the fracture healing process. The reason for the results of no remarkable changes of serum intact $\mathrm{OC}$ during the study period is that serum intact OC might be less sensitive to both drugs than serum BAP as an indicator for bone turnover. No previous reports have demonstrated the changes of intact $\mathrm{OC}$ during alendronate or raloxifene therapy after hip fracture. Further study will be necessary for the use of serum intact $\mathrm{OC}$ for monitoring the effect of drugs.

Patients with hip fracture are candidates for anti-osteoporosis drug intervention according to the guidelines for osteoporosis ${ }^{3)}$. Treatment with anti-osteoporosis drugs within 6 months after hip fracture has improved over the past 10 years, but some authors have reported that anti-osteoporosis drugs are administered to only $6-10 \%$ of all hip fracture patients ${ }^{26,27)}$. Such a low rate of drug intervention for patients with hip fracture is not only due to the responsible doctors not prescribing such medicines but also because of the lower level of patient compliance and persistence with anti-osteoporosis drugs. In this study, almost half of all the patients who entered the study dropped out. In particular, more patients in group ALN dropped out than in group RLX. No cases were withdrawn due to side effect of alendronate. The reason for the group difference in dropout rate might be that more patients entered the study in group ALN than in group RLX. Some patients did not visit our outpatient ward every one to three months after discharge as we directed, although we emphasized the importance of such visits to the patients and their families for continuing administration of their particular drug to prevent bone loss. Discontinuance of medicine is the most serious problem hampering prevention of bone loss after hip fracture. According to Bell et al., BMD measurement is not necessary during the 3 to 5 years after bisphosphonate administration in patients with osteoporosis since misreadings may occur ${ }^{28)}$. However, we believe the most important thing is keeping the level of patient compliance with anti-osteoporosis drugs high, since there is evidence that both alendronate and raloxifene prevent fragile fractures. How can we keep patient compliance high? In the present study, trochanter or total BMD of the contralateral hip increased in the short term. If responsible doctors show data for the trochanter or total hip BMD to their hip fracture patients, patient compliance with anti-osteoporosis drugs may well improve. In Japan, BMDs for lumbar spine and femoral neck are mostly used to monitor changes during anti-osteoporosis therapy. We suggest that trochanter and total BMDs of the proximal femur should also be monitored after hip fracture during anti-osteoporosis drug therapy.

One of the limitations of this study is the smaller than expected sample size due to the surprisingly high dropout rate. Therefore, we focused not only on the effect of alendronate or raloxifene on BMDs but also the reasons for dropping out. Another limitation is that control or placebo cases were not available in this study. In our previous study, we monitored the spine and proximal femur in patients with hip fractures without drug intervention in the same design as the present study ${ }^{24)}$. That study found that BMDs of the L2-4 spine and 4 regions of the proximal femur decreased during 9 months without anti-osteoporosis drug intervention after hip fracture.

In conclusion, both alendronate and raloxifene have a favorable effect on trochanter and total BMDs of the contralateral proximal femur in the early period after operation for patients with hip fractures.

\section{Acknowledgment}

We wish to give special thanks to Miss Ayako Okamoto for measuring biochemical markers.

\section{References}

1. Schroder HM, Petersen KK, Erlandsen M. Occurrence and incidence of the second hip fractute. Clin Orthop 1993; 289: 166-169.[Medline]

2. Boston DA. Bilateral fractures of the femoral neck. Injury 1983; 14: 207-210.[Medline] [CrossRef]

3. Orimo H. Japanese Guideline for Prevention and Treatment of Osteoporosis. Life Science 2006.

4. Black DM, Cummings SR, Karpf DB, et al. Randomised trial of effect of alendronate on risk of fracture in women with existing vertebral fractures. Lancet 1996; 348: 1535-1541. [Medline] [CrossRef]

5. Cummings SR, Black DM, Thompson DE, et al. Effect of alendronate on risk of fracture in women with low bone density but without vertebral fractures: results from the fracture intervention trial. JAMA 1998; 280: 2077-2082.[Medline] [CrossRef]

6. Kushida K, Shiraki M, Nakamura T, et al. Alendronate reduced vertebral fracture risk in postmenopausal Japanese women with osteoporosis: a 3-year follow-up study. J Bone Miner Metab 2004; 22: 462-468.[Medline] [CrossRef]

7. Ensrud KE, Stock JL, Barrett-Connor E, et al. Effects of raloxifene on fracture risk in postmenopausal women: The raloxifene use for the heart trial. J Bone Miner Res 2008; 23: 112-120.[Medline] [CrossRef]

8. Delmas PD, Ensrud KE, Adachi JD, et al. Efficacy of raloxifene on vertebral fracture risk in postmenopausal women with osteoporosis: four-year results from a randomized clinical trial. J Clin Endocrinol Metab 2002; 87: 3609-3617.[Medline] [CrossRef]

9. Delmas PD, Genant HK, Crans GG, et al. Severity of prevalent vertebral fractures and the risk of subsequent vertebral 
and nonvertebral fractures: results from the MORE trial. Bone 2003; 33: 522-532.[Medline] [CrossRef]

10. Gardner MJ, Flik KR, Mooar P, et al. Improvement in the undertreatment of osteoporosis following hip fracture. JBJS 2005; 84-A(8): 1342-1348.[Medline]

11. Cadarette SM, Katz JN, Brookhart MA, et al. Trends in drug prescribing for osteoporosis after hip fracture, 1995-2004. J Rheumatol 2008; 35: 319-326.[Medline]

12. Feldstein AC, Nichols GA, Elmer PJ, et al. Older women with fractures: patients falling through the cracks of guideline-recommended osteoporosis screening and treatment. JBJS 2003; 85-A(12): 2294-2302.[Medline]

13. Hajcsar EE, Hawker G, Boboch ER. Investigation and treatment of osteoporosis in patients with fragility fractures. CMAJ 2000; 163: 819-822.[Medline]

14. Carnevale V, Nieddu L, Romagnoli E, et al. Osteoporosis intervention in ambulatory patients with previous hip fracture: a multicentric, nationwide Italian survey. Osteoporos Int 2006; 17: 478-483.[Medline] [CrossRef]

15. Liel Y, Castel H, Bonneth DY. Impact of subsidizing effective anti-osteoporosis drugs on compliance with management guidelines in patients following low-impact fractures. Osteoporos Int 2003; 14: 490-495.[Medline] [CrossRef]

16. Gomez B, Ardakani S, Ju Julia, Jenkins D, et al. Monoclonal antibody assay for measuring bone-specific alkaline phosphatase activity in serum. Clin Chem 1995; 41: 1560-1566.[Medline]

17. Hanson DA, Weis MAE, Bollen AM, et al. A specific immunoassay for monitoring human bone resorption: quantitation of type I collagen cross-linked N-telopeptides in urine. J Bone Miner Res 1992; 7: 1251-1258.[Medline] [CrossRef]

18. Dirschl DR, Henderson RC, Oakley WC. Accelerated bone mineral loss following a hip fracture: a prospective longitudi- nal study. Bone 1997; 20: 79-82.[Medline] [CrossRef]

19. Dirschl DR, Piedrahita L, Henderson RC. Bone mineral density 6 years after a hip fracture: A prospective, longitudinal study. Bone 2000; 26: 95-98.[Medline] [CrossRef]

20. Yu-Yahiro J, Michael RH, Dubin NH, et al. Serum and urine markers of bone metabolism during the year after hip fracture. JAGS 2001; 49: 877-883.[Medline] [CrossRef]

21. Karachelios T, Lyritis GP, Kaloudis J, et al. The effect of calcitonin on acute bone loss after peritrochanteric fractures. JBJS 2004; 86-B: 350-358.

22. Hedstrom M, Sjoberg K, Svensson J, et al. Changes on biochemical markers of bone metabolism and BMD during the first year after a hip fracture. Acta Orthop Scand 2001; 72: 248-251.[Medline] [CrossRef]

23. Cecilia D, Jodar E, Fernandez C, et al. Effect of alendronate in elderly patients after low trauma hip fracture repair. Osteoporos Int 2009; 20: 903-910.[Medline] [CrossRef]

24. Ohishi T, Takahashi M, Nagano A. Vitamin $\mathrm{K}_{2}$ and etidronate therapy in the early period after hip fracture. JRM 2007; 2: 105-115.

25. Ohishi T, Takahashi M, Kushida K, et al. Changes of biochemical markers during fracture healing. Arch Orthop Traum Surg 1998; 118: 126-130.[Medline] [CrossRef]

26. Juby AG, De Geus-Wenceslau CM. Evaluation of osteoporosis treatment in seniors after hip fracture. Osteoporos Int 2002; 13: 205-210.[Medline] [CrossRef]

27. Rabenda V, Mertens R, Fabri V, et al. Adherence to bisphosphonates therapy and hip fracture risk in osteoporotic women. Osteoporos Int 2008; 19: 811-818.[Medline] [CrossRef]

28. Bell KJL, Hayen A, Macaskill P, et al. Value of routine monitoring of bone mineral density after starting bisphosphonate treatment: secondary analysis of trial data. BMJ 2009; 338: b2266.[Medline] [CrossRef] 